\title{
Erratum: Viscosity and real-space molecular motion of water: Observation with inelastic x-ray scattering [Phys. Rev. E 98, 022604 (2018)]
}

\author{
Yuya Shinohara, Wojciech Dmowski, Takuya Iwashita, Bin Wu, Daisuke Ishikawa, \\ Alfred Q. R. Baron, and Takeshi Egami 10
}

(Received 29 August 2019; published 30 September 2019)

DOI: 10.1103/PhysRevE.100.039904

We have found that we inadvertently used incorrect time-axis values for the Van Hove functions calculated from the inelastic X-ray scattering (IXS) experiment; a factor of 2 larger than the correct values. This influences the timescale in Figs. 1, 2 (left), and 4, and the parameter of decaying behaviors in Table I. The time-axis values for the Van Hove function calculated from the molecular dynamics (MD) simulation is correct and unchanged. None of the conclusions and discussion in the original paper are

(a)
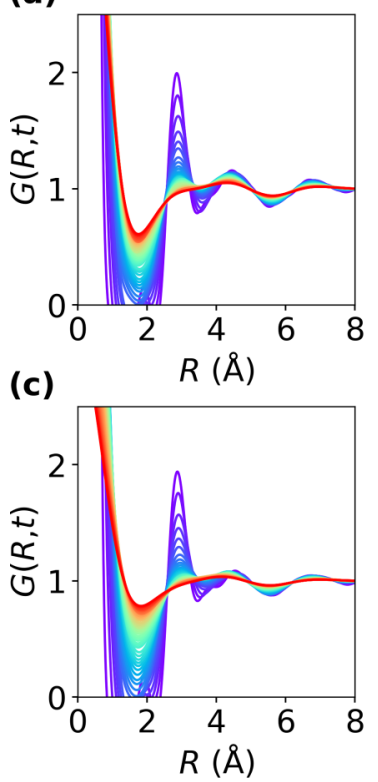

(b)

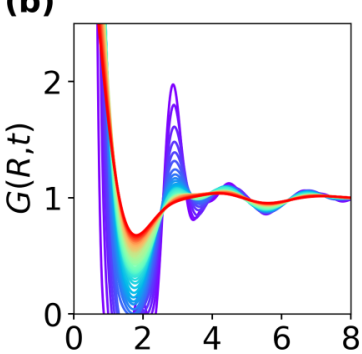

(d) $\quad R(\AA)$

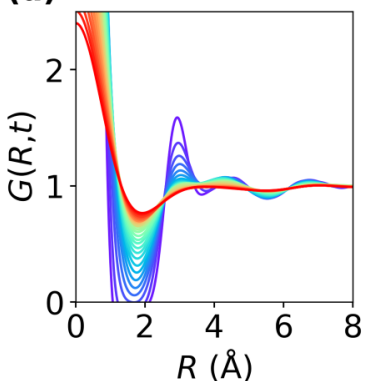

(e)

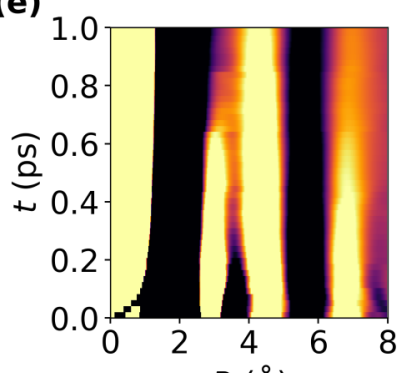

(g)

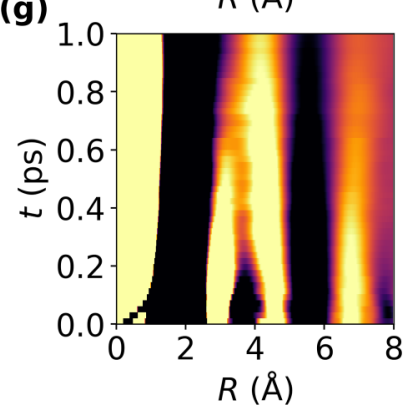

(f)

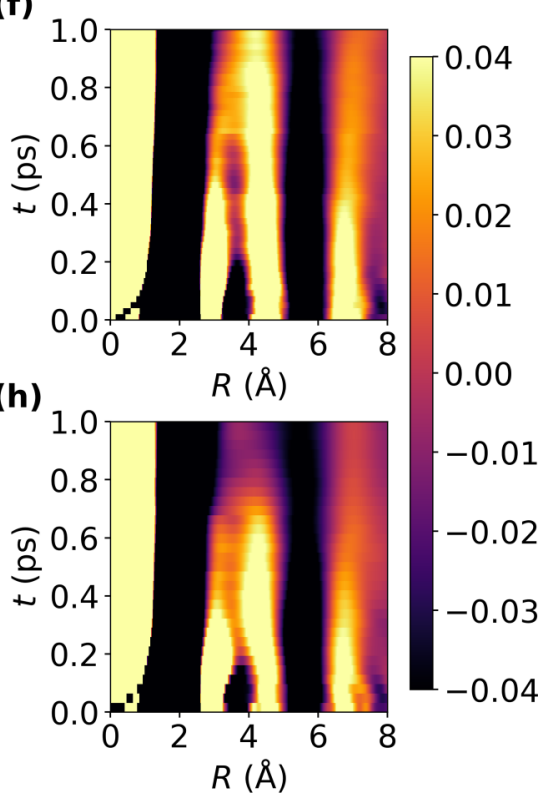

FIG. 1. Van Hove function of water at ambient temperatures obtained from IXS results. (a)-(d) One-dimensional profiles at $0.06 \mathrm{ps}<t<$ 1 ps. The arrows indicate the direction of temporal evolution. (e)-(h) Intensity map of $G(R, t)-1$. Note that a narrow range is used to highlight small changes in the second and third peaks. Temperature of the water was $285 \mathrm{~K}$ (a), (e), $295 \mathrm{~K}$ (b), (f), $310 \mathrm{~K}$ (c), (g), and $318 \mathrm{~K}$ (d), (h).
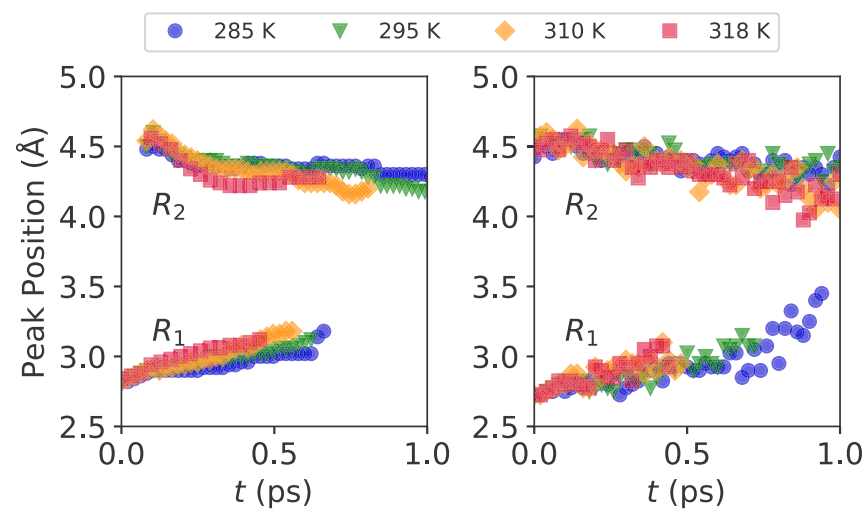

FIG. 2. Temporal changes in the first and the second peak positions, $R_{1}$ and $R_{2}$, of the Van Hove functions calculated from (left) the IXS data and (right) the MD calculation. The temperature of the sample shown in the inset. 
(a)

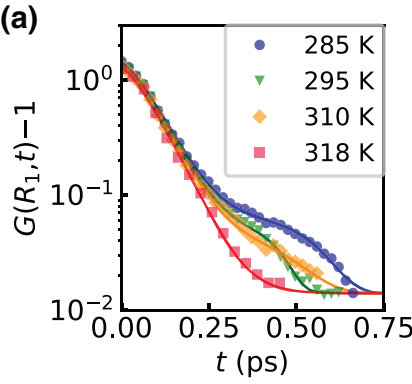

(c)

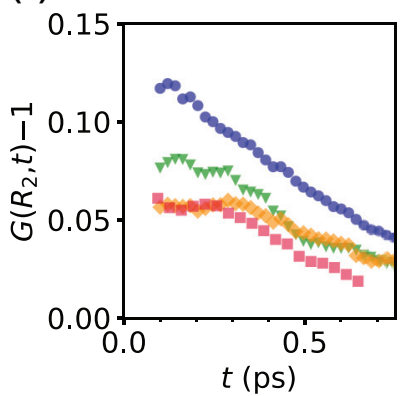

(b)

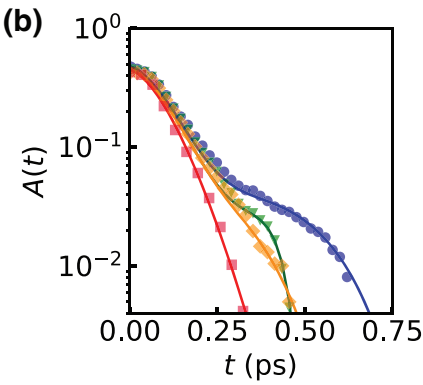

(d)

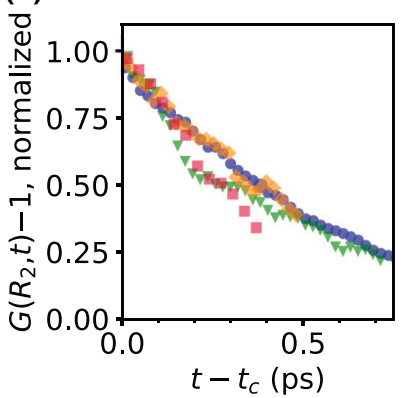

FIG. 4. Temporal changes in (a) the height of the first peak, $G(R, t)-1$, and (b) the area of the first peak, $A(t)$, calculated from the IXS results. Lines are the result of fitting using two compressed exponential functions. Temperatures are shown in the inset of the left panel. (c) Temporal change in the height of the second peak, calculated from the IXS results, and (d) its normalized intensity dependence on the reduced time.

affected, except for the discrepancies between the values of relaxation time determined by the IXS and the MD. In the original paper, the values from IXS were almost twice as large as those from MD. The discrepancies were canceled out by using the correct timescale for the IXS. This does not affect our conclusion in the original paper but rather opens the possibility of wider use of the Van Hove function approach with the MD simulation for identifying the correlated dynamics in liquid.

The necessary corrections are as follows:

(1) The last sentences in the right column on p. 2 should read: "The first peak entirely decays after $t=0.4-0.6 \mathrm{ps,} \mathrm{depending}$ on the sample temperature. At higher temperatures ( 310 and $318 \mathrm{~K}$ ), the position of the second maximum shifts rather sharply up to 0.3 ps." The final sentences in the first paragraph of Sec. III B on p. 3 should read "The decay time of the first component $\tau_{1}$ also remains unchanged with a temperature at $0.13 \mathrm{ps}$, and the value of $A_{1}$ is also insensitive to the sample temperature. However, the decay time of the second component $\tau_{2}$ varies with temperature from 0.4 to 0.6 ps."

(2) The following sentence, then, near the end of Sec. II B on p. 4 should be deleted: "On the other hand, the values of the relaxation times are significantly different from the IXS results when the results of MD simulation were fitted with Eq. (4)."

(3) The text on p. 5, right column, third paragraph should be changed to "The movement of the second peak position is fast for $T=310$ and $318 \mathrm{~K}$ up to $t=0.250 \mathrm{ps}$, followed by small changes around $4.25 \AA$, whereas it is monotonic for $T=285$ and 295 K." The text on p. 5, at the start of the first paragraph of Sec. IV A should be changed to "We surmise that the first decay originates from the ballistic motions of molecules, observed for a short time $(0.15 \mathrm{ps})$ before they hit the cage of the neighbors."

(4) The following sentences on p. 6, left column, lines 18-22, should be deleted: "As shown in Fig. 5, MD simulation underestimates $\tau_{2}$ by a factor of 2 . Even though the MD results are qualitatively similar to the experimental results, such qualitative differences are alarming." Also, the following text on p. 7, right column, lines 11-14, should be deleted: "The estimated value of $\tau_{\mathrm{LC}}$ at $295 \mathrm{~K}, \sim 0.86 \mathrm{ps}$ is longer than the estimate in the previous study $\sim 0.4 \mathrm{ps}$ [35]. This discrepancy originates from the much-improved statistics of the present IXS data." On p. 8, right column, at the end of the Concluding Remarks, the following sentences should be deleted: "Classical interatomic potentials for water were capable of describing the

TABLE I. Fitting results of the first peak area, $A(t)$, of the Van Hove function obtained by IXS.

\begin{tabular}{lllllll}
\hline \hline & $\tau_{1} / \mathrm{ps}$ & $\tau_{2} / \mathrm{ps}$ & $A_{1}$ & $A_{2}$ & $\gamma_{1}$ & $\gamma_{2}$ \\
\hline $285 \mathrm{~K}$ & 0.12 & 0.57 & 0.44 & 0.036 & 3.4 \\
$295 \mathrm{~K}$ & 0.12 & 0.43 & 0.45 & 0.018 & $1.57^{\mathrm{a}}$ & $1.57^{\mathrm{a}}$ \\
$310 \mathrm{~K}$ & 0.12 & 0.4 & 0.42 & 0.026 & $1.57^{\mathrm{a}}$ & 1.57 \\
$318 \mathrm{~K}$ & 0.12 & & 0.45 & & 4.1 \\
\hline \hline
\end{tabular}

\footnotetext{
${ }^{\text {a}}$ Fixed.
} 


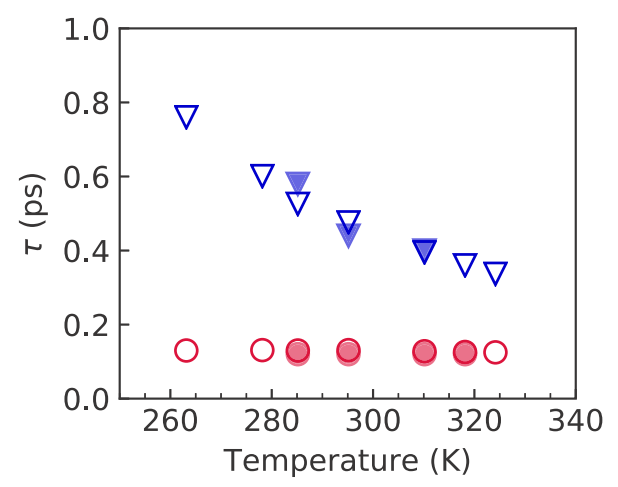

FIG. 5. Temperature dependence of $\tau_{1}$ (circles) and $\tau_{2}$ (triangles) obtained from IXS (closed symbols) and MD (open symbols).

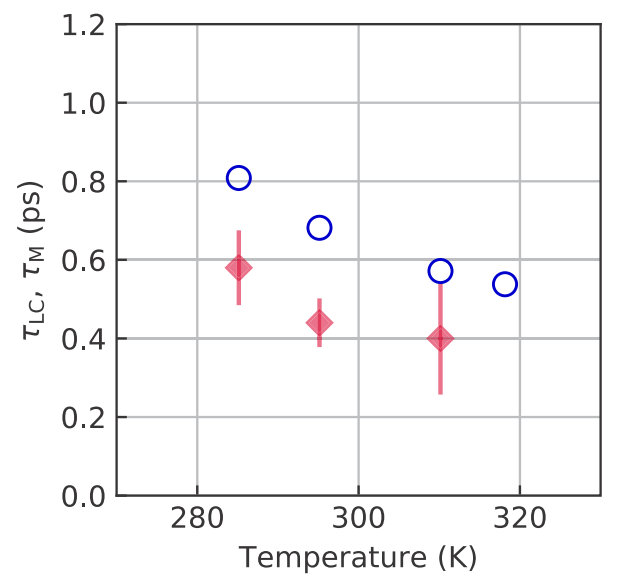

FIG. 9. Temperature dependence of the Maxwell relaxation time, $\tau_{M}$ (circles) and the excitation time, $\tau_{\mathrm{LC}}$ (diamonds), derived from the IXS measurement by $\tau_{\mathrm{LC}} \approx \tau_{2}$. The Maxwell relaxation time is calculated from the viscosity [54] and shear modulus, $G_{\infty}$ from ultrasonic viscosity measurement [55]. The error bars for $\tau_{\mathrm{LC}}$ correspond to the two standard deviations of the mean for $\tau_{2}$.

local dynamics qualitatively, but there are serious discrepancies, indicating the need of fully quantum-mechanical calculations for describing the dynamics of the hydrogen bond." Indeed, the agreements between the IXS experiments and the MD and those between this paper and Ref. [35] are excellent after the correction.

(5) Timescales of Figs. 1, 2, and 4 should be changed, and the fitting result in Figs. 5 and 9 needs updates. The complete Figs. 1, 2, 4, 5, and 9 are reprinted here.

[35] T. Iwashita, W. Bin, W.-R. Chen, S. Tsutsui, A. Q. R. Baron, and T. Egami, Seeing real-space dynamics of liquid water through inelastic X-ray scattering, Sci. Adv. 3, e1603079 (2017).

[54] L. Korson, W. Drost-Hanse, and F. J. Millero, Viscosity of water at various temperatures, J. Phys. Chem. 73, 34 (1969).

[55] W. M. Slie, A. R. Donfor, and T. A. Litovitz, Ultrasonic shear and longitudinal measurements in aqueous glycerol, J. Chem. Phys. 44, 3712 (1966). 